\title{
Deterministic propagation of vortex-antivortex pairs in magnetic trilayers
}

A. Hierro-Rodriguez, C. Quirós, A. Sorrentino, R. Valcárcel, I. Estébanez, L. M. Alvarez-Prado, J. I. Martín, J. M. Alameda, E. Pereiro, M. Vélez, and S. Ferrer

Citation: Appl. Phys. Lett. 110, 262402 (2017); doi: 10.1063/1.4984898

View online: https://doi.org/10.1063/1.4984898

View Table of Contents: http://aip.scitation.org/toc/apl/110/26

Published by the American Institute of Physics

\section{Articles you may be interested in}

Vortex circulation patterns in planar microdisk arrays

Applied Physics Letters 110, 262406 (2017); 10.1063/1.4990990

Spin Hall magnetoresistance in an antiferromagnetic magnetoelectric $\mathrm{Cr}_{2} \mathrm{O}_{3}$ /heavy-metal W heterostructure Applied Physics Letters 110, 262401 (2017); 10.1063/1.4989680

Making the Dzyaloshinskii-Moriya interaction visible Applied Physics Letters 110, 242402 (2017); 10.1063/1.4985649

Electrical switching of the magnetic vortex circulation in artificial multiferroic structure of $\mathrm{Co} / \mathrm{Cu} / \mathrm{PMN}-\mathrm{PT}(011)$ Applied Physics Letters 110, 262405 (2017); 10.1063/1.4990987

Subnanosecond microwave-assisted magnetization switching in a circularly polarized microwave magnetic field Applied Physics Letters 110, 262403 (2017); 10.1063/1.4986483

Asymmetrical domain wall propagation in bifurcated PMA wire structure due to the Dzyaloshinskii-Moriya interaction

Applied Physics Letters 110, 232402 (2017); 10.1063/1.4984750

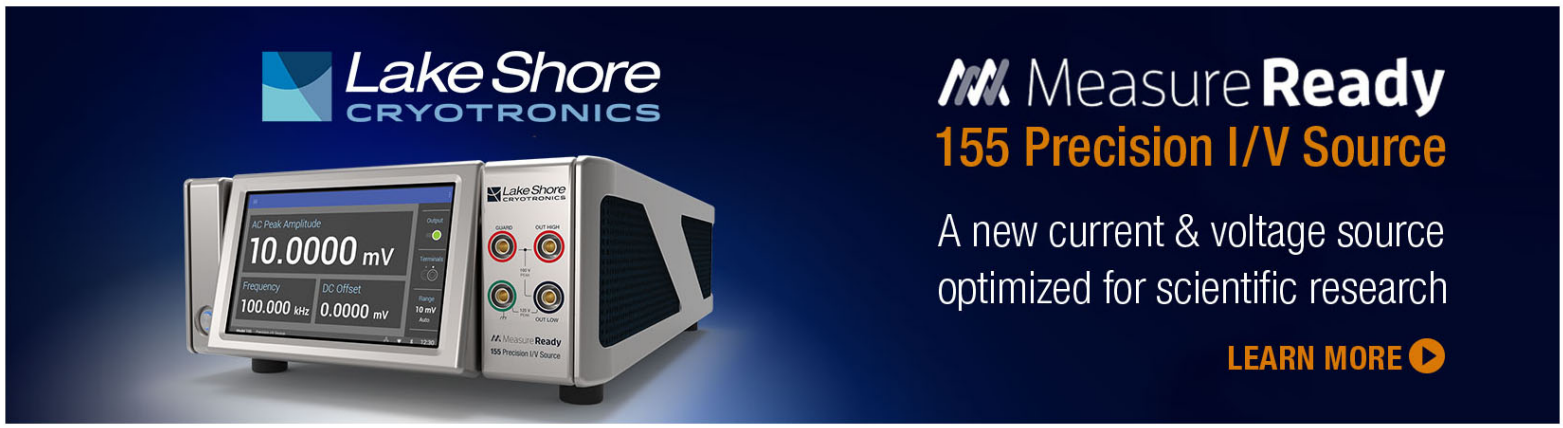




\title{
Deterministic propagation of vortex-antivortex pairs in magnetic trilayers
}

\author{
A. Hierro-Rodriguez, ${ }^{1}$ C. Quirós, ${ }^{2,3}$ A. Sorrentino, ${ }^{4}$ R. Valcárcel, ${ }^{4}$ I. Estébanez, ${ }^{2}$ \\ L. M. Alvarez-Prado, ${ }^{2,3}$ J. I. Martín, ${ }^{2,3}$ J. M. Alameda, ${ }^{2,3}$ E. Pereiro, ${ }^{4}$ M. Vélez, ${ }^{2,3}$ \\ and S. Ferrer ${ }^{4}$ \\ ${ }^{1}$ IN-IFIMUP, Departamento de Física e Astronomia, Faculdade de Ciencias, Universidade do Porto, \\ 4169-007 Porto, Portugal \\ ${ }^{2}$ Departamento de Física, Universidad de Oviedo, 33007 Oviedo, Spain \\ ${ }^{3}$ Centro de Investigación en Nanomateriales y Nanotecnología, CINN (CSIC - Universidad de Oviedo), \\ 33940 El Entrego, Spain \\ ${ }^{4}$ ALBA Synchrotron, 08290 Cerdanyola del Vallès, Spain
}

(Received 28 April 2017; accepted 20 May 2017; published online 27 June 2017)

\begin{abstract}
Thin perpendicular magnetic anisotropy films between two soft ferromagnetic layers have the nuclei for magnetization inversion at the bifurcations of their characteristic stripe domain pattern. The inverted nuclei induce vortex-antivortex pairs in the soft magnetic layers that exhibit a correlated motion extending several $\mu \mathrm{m}$ along the magnetic stripes during magnetization reversal. The sense of motion is completely determined by the topology of the magnetic bifurcations causing vortex-antivortex pairs to propagate in opposite senses depending on their polarities. This is a robust effect that might have practical applications. These findings are based on X-ray microscopy and micromagnetic calculations. Published by AIP Publishing.
\end{abstract}

[http://dx.doi.org/10.1063/1.4984898]

Magnetic vortex-antivortex (V-AV) pairs have a topology characterized by skyrmion number $\mathrm{Q}=0$ or 1 depending on their relative polarity. ${ }^{1}$ They emerge in a variety of dynamical processes such as vortex core reversal in magnetic dots, ${ }^{2,3}$ Walker breakdown in domain wall propagation along magnetic nanowires, ${ }^{4,5}$ or in phase control of spin torque nano-oscillators. ${ }^{6}$ Also, different memory schemes have been proposed based on the propagation of vortices by the nanocontact stray field ${ }^{7}$ or vortex-antivortex pairs in permalloy nanowires. ${ }^{8}$

Magnetic textures with $\mathrm{Q}=0$ (bubbles), ${ }^{9} \mathrm{Q}=1 / 2$ (merons), ${ }^{10}$ and $\mathrm{Q}=1$ (dipolar skyrmions) ${ }^{9}$ have been nucleated in a controlled way in RE-TM multilayers and alloys $(\mathrm{RE}=\mathrm{Nd}, \mathrm{Gd} ; \mathrm{TM}=\mathrm{Co}, \mathrm{Fe})$ by properly adjusting material parameters such as out-of-plane magnetic anisotropy $\left(\mathrm{K}_{\mathrm{N}}\right)$, exchange stiffness, $(A)$ and saturation magnetization $\left(\mathrm{M}_{\mathrm{S}}\right)^{9}$ or by surface patterning. ${ }^{11}$ In these materials, when $\mathrm{K}_{\mathrm{N}}<1 / 2$ $\mu_{0} \mathrm{M}_{\mathrm{S}}{ }^{2}$ [named hereafter weak perpendicular magnetic anisotropy (wPMA)], the magnetization is arranged in periodic stripe domain patterns along an in-plane direction defined by the last saturating field. ${ }^{12}$ This stripe pattern is locked in place by rotatable anisotropy and reconfigurable through magnetic history. ${ }^{11,13}$ These two properties provide the basis for tunable magnetic devices ${ }^{14}$ and have been used for the guided propagation of superconducting vortices in magnetic/superconducting hybrids. ${ }^{15,16}$

Stray fields and exchange interactions can be used to imprint complex magnetic textures as bubbles or skyrmions on soft magnetic layers in multilayers combining perpendicular magnetic anisotropy (PMA) and in-plane anisotropy materials. ${ }^{10,17,18}$ For example, in NiFe/NdCo(wPMA)/GdCo multilayers, the stripe pattern of the central $\mathrm{NdCo}$ layer is replicated at the top NiFe and bottom GdCo layers, ${ }^{19}$ including linear defects in the stripe pattern such as dislocations (bifurcations and endpoints). At the onset of in-plane magnetization reversal, magnetic textures such as $\mathrm{Q}=1$ Bloch points (BP) and $\mathrm{Q}=1 / 2$ merons appear in the central $\mathrm{NdCo}$ layer and drive the nucleation of reversed nuclei in the permalloy layer. ${ }^{19}$ Then, the geometry of the stripe pattern and localized asymmetries of the stray field may be used to confine and guide the propagation of these reversed domains in the permalloy layer, without physical patterning.

Here, we report on the propagation of magnetic vortexantivortex pairs with opposite polarities (i.e., $\mathrm{Q}=1$ ) during the magnetization reversal process of $\mathrm{NiFe} / \mathrm{NdCo} / \mathrm{GdCo}$ multilayers under the effect of pulsed magnetic fields. Element resolved magnetic transmission x-ray microscopy and micromagnetic simulations show that preferred nucleation of reversed magnetic domains occurs at dislocations in the stripe pattern. The propagation in the permalloy layer of vortex-antivortex pairs from these dislocations is guided by the stray field of a pair of up/down stripe domains in the NdCo layer underneath, which results in a deterministic and predictable sense of motion of both singularities.

Magnetic trilayers consisting on $40 \mathrm{~nm} \mathrm{Ni}{ }_{80} \mathrm{Fe}_{20} / 60 \mathrm{~nm}$ $\mathrm{Nd}_{16} \mathrm{Co}_{84} / 40 \mathrm{~nm} \mathrm{Gd}{ }_{12} \mathrm{Co}_{88}$ [Fig. 1(a)] were grown on $50 \mathrm{~nm}$ thick $\mathrm{Si}_{3} \mathrm{~N}_{4}$ membranes by dc magnetron sputtering as reported previously. ${ }^{19} \mathrm{Gd}_{12} \mathrm{Co}_{88}$ is a ferrimagnetic alloy with $\mathrm{M}_{\mathrm{S}}=6 \times 10^{5} \mathrm{~A} / \mathrm{m}$ and in-plane uniaxial anisotropy ${ }^{20} \mathrm{~K}_{\mathrm{u}}$ $=4.6 \times 10^{3} \mathrm{~J} / \mathrm{m}^{3} . \mathrm{Nd}_{16} \mathrm{Co}_{84}$ is an amorphous ferromagnetic ${ }^{11}$ alloy with $\mathrm{M}_{\mathrm{S}}=7 \times 10^{5} \mathrm{~A} / \mathrm{m}$ and $\mathrm{PMA} \mathrm{K}_{\mathrm{N}} \approx 10^{5} \mathrm{~J} / \mathrm{m}^{3}$. Permalloy $\mathrm{Ni}_{80} \mathrm{Fe}_{20}$ has $\mathrm{M}_{\mathrm{S}}=8.5 \times 10^{5} \mathrm{~A} / \mathrm{m}$ and in-plane $\mathrm{K}_{\mathrm{u}}=850 \mathrm{~J} / \mathrm{m}^{3}$. Strains during growth may induce a weak PMA in the permalloy layer ${ }^{15}$ of the order $\mathrm{K}_{\mathrm{N}} \approx 10^{4} \mathrm{~J} / \mathrm{m}^{3}$, too low to induce out-of-plane domains in the samples investigated.

As described previously, ${ }^{10,19}$ element specific magnetic contrast imaging can be achieved using the transmission microscope of the Mistral Beamline at Alba synchrotron ${ }^{21,22}$ by tuning the circularly polarized X-rays to the appropriate 

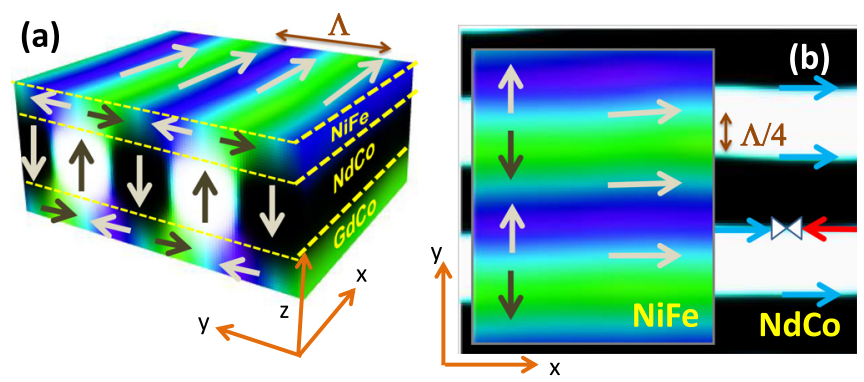

FIG. 1. (a) Magnetization configuration in a $40 \mathrm{~nm} \mathrm{NiFe} / 60 \mathrm{~nm} \mathrm{NdCo} / 40 \mathrm{~nm}$ GdCo trilayer at remanence (lateral view): the stray field of up/down stripe domains in the central NdCo layer creates a pattern of closure domains in top/bottom NiFe/GdCo layers. The stripe domain period $\Lambda$ is indicated by the double arrow. (b) Sketch of the relative position of up/down stripe domains in the NdCo central layer (white/black indicates the $+\mathrm{M}_{\mathrm{z}} /-\mathrm{M}_{\mathrm{z}}$ domains) and the closure structure in the NiFe top layer (top view). Note that the lines of maximum $\mathrm{M}_{\mathrm{x}}$ (horizontal arrows) are shifted by $\Lambda / 4$ between NdCo and NiFe layers. A head-to-head Bloch point (double triangle) at a domain wall of the central layer is also sketched.

atomic absorption energy of an element. Here, we report images of the magnetization of the upper permalloy layer acquired at the $\mathrm{L}_{3} \mathrm{Fe}$ absorption energy. The X-ray angle of incidence was set at $\theta=30^{\circ}$ from the film normal, in order to have sensitivity to $\mathrm{M}_{\mathrm{x}}$ and $\mathrm{M}_{\mathrm{z}}$ magnetization components. The sample was first imaged at remanence after saturating it with an in-plane field $\mu_{0} \mathrm{H}_{\mathrm{x}}=225 \mathrm{mT}$, and then, negative $\mathrm{H}_{\mathrm{x}}$ pulses of $20 \mu$ s duration and variable amplitude were applied in-situ to monitor the magnetization reversal.

Magnetic domain images were compared with micromagnetic simulations of magnetization reversal. Calculations were performed using the finite difference ${ }^{23}$ code $\operatorname{MuMax}^{3}$ with a discretization into cells of dimensions of $5 \times 5 \times 3 \mathrm{~nm}^{3}$ for a total of $3840 \times 3840 \times 141 \mathrm{~nm}^{3}$ and material parameters for each sublayer obtained from the magnetic characterization. ${ }^{19}$ MuView code was used for visualization. ${ }^{24}$ Simulated X-ray image contrasts were obtained from the calculated micromagnetic configurations by evaluating the contribution to the dichroic absorption factor of each cell along the X-ray beam path (see the details in the supplementary material).

Figure 1 shows a sketch of the simulated stripe domain pattern in the trilayer at remanence after saturating it with an in-plane field $\mu_{0} \mathrm{H}_{\mathrm{x}}=225 \mathrm{mT}$. In the central NdCo layer, the magnetization is organized in parallel up/down $\pm \mathrm{M}_{\mathrm{z}}$ domains with period $\Lambda$, separated by Bloch walls in which in-plane magnetization $\mathrm{M}_{\mathrm{x}}$ is maximum. Due to topological restrictions, ${ }^{25}$ in-plane magnetization reversal occurs by the propagation of head-to-head $(\mathrm{Q}=-1)$ or tail-to-tail $(\mathrm{Q}=+1)$ Bloch points moving along these Bloch walls [Fig. 1(b)]. In the top NiFe layer, the magnetization follows the closure domain pattern (white/black arrows along the y axis) induced by the $M_{z}$ magnetization in the central layer. Regions of $M_{y}$ $>0$ and $M_{y}<0$ are separated by Neel walls in which $M_{x}$ is maximum. Additionally, the top layer has $M_{x}$ and $M_{z}$ magnetization contributions imprinted by the middle layer. The pattern of closure domains across the multilayer thickness originates a shift of the Neel walls $\left(\mathrm{M}_{\mathrm{x}}\right.$ maximum) by $\Lambda / 4$ in the $\mathrm{NiFe}$ layer, which results in the arrangement shown in Fig. 1(b). As it will be clear in what follows, this $\Lambda / 4$ shift is essential for the lateral confinement of vortex-antivortex pairs in the NiFe layer during magnetization reversal.
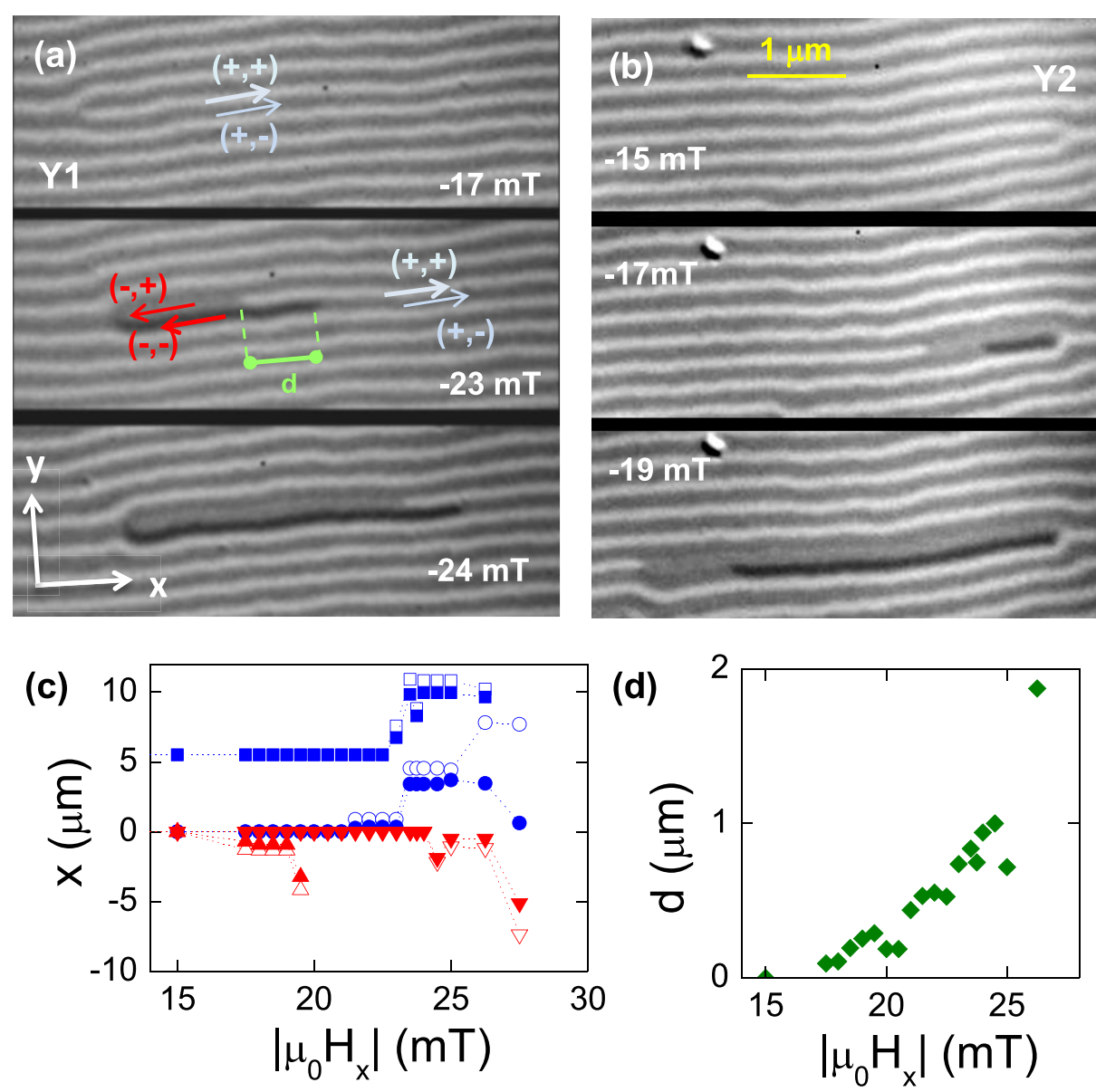

FIG. 2. (a) and (b) Magnetic images of the propagation of reversed domains in the NiFe layer under a sequence of $\mathrm{H}_{\mathrm{x}}$ pulses, as indicated in each panel. The signs of $\left(\mathrm{M}_{\mathrm{x}}, \mathrm{M}_{\mathrm{z}}\right)$ magnetizations at different locations are indicated and also the separation $d$ of the reversed branches. (c) Length of the reversed stripes vs. field amplitude for branches (open symbols) and central stripes (filled symbols). The reversed central stripes have either $M_{z}>0$ (squares and circles) or $\mathrm{M}_{\mathrm{z}}<0$ (triangles). Positive/negative $x$ corresponds to the right/left propagation of the reversed domain. The pair of $(\square, \square)$ curves has been vertically shifted for clarity. (d) Average separation between the inverted branch and the central stripe [ $d$ in panel (a)] vs. field amplitude. 
Figure 2 shows two sequences of images from different regions in the $\mathrm{NiFe}$ layer, acquired after applying reversed field pulses of increasing amplitude. In the initial state, the images display a pattern of bright/dark parallel stripes oriented along the $x$ direction of period $\Lambda=250 \mathrm{~nm}$. Due to the oblique incidence of the $\mathrm{X}$-rays and the sample mounting geometry, the image contrast depends on $\mathrm{M}_{\mathrm{x}}$ and $\mathrm{M}_{\mathrm{z}}$ and is not sensitive to $\mathrm{M}_{\mathrm{y}}$. As indicated by arrows in Fig. 2(a), parallel stripes with $\left(\mathrm{M}_{\mathrm{x}}, \mathrm{M}_{\mathrm{z}}\right)$ having signs $(+,+)$ and $(+,-)$ run parallel to the $x$ axis. Magnetic defects in the pattern (dislocations) are indicated by $\mathrm{Y} 1$ and $\mathrm{Y} 2$ and consist of a bifurcation and an end-point in the middle of the bifurcation. The application of reversed field pulses originates the appearance of two additional contrast levels that can be assigned to $(-,+)$ and $(-,-)$ magnetizations. ${ }^{19}$ For example, dislocation $\mathrm{Y} 1$ in Fig. 2(a) is composed of a dark stripe bifurcation and a white end-point in the initial state, but after a $\mu_{0} \mathrm{H}_{\mathrm{x}}=-23 \mathrm{mT}$ field pulse, the magnetic contrast at the lower bifurcation branch and the central stripe becomes darker, indicating $\mathrm{M}_{\mathrm{x}}$ reversal.

Upon increasing the pulsed field amplitude, these reversed regions expand towards the right of the image (positive $x$ ), propagating along the lines defined by the stripe pattern. The sequence shown in Fig. 2(b) starts at Y2, a dislocation composed of a white stripe bifurcation and a dark central endpoint, with the reversed domain expanding towards the negative $x$ direction. The lengths of reversed domains upon increasing the field amplitude are plotted in Fig. 2(c). In a typical case, e.g., circles in Fig. 2(c), the reversed branch $(O)$ and reversed central stripe $(0)$ expand in a correlative fashion at low fields (from $21 \mathrm{mT}$ to $25 \mathrm{mT}$ ) for about $5 \mu \mathrm{m}$ along the stripe pattern, with a slightly longer reversed branch (ca. $0.5-1 \mu \mathrm{m})$. At larger fields, this motion becomes uncoupled and, even, a backward jump is observed in the central stripe (๖) at $27 \mathrm{mT}$.

Some general features can be extracted from the analysis of the propagation of 17 different reversed domains observed in our measurements: (i) reversed domains are guided by the lines defined by the local stripe pattern, (ii) the preferred propagation sense is towards the left (negative $x$ ) for dark central stripes [as in Fig. 2(b)] and towards the right (positive $x$ ) for bright central stripes [Fig. 2(a)], (iii) the observed propagation occurs by discrete jumps typical for an activated process during the applied field pulse that relaxes to local pinning sites when the sample is imaged at remanence [short backwards jumps are also observed in Fig. 2(c)], (iv) at low fields, a correlated motion of the reversed branch and central stripe is observed with the reversed branch moving ahead in $90 \%$ of the cases, (v) average separation $d$ between the reversed branch/central stripe increases with the applied field amplitude [see Fig. 2(d)] until they become uncoupled for distances above ca. $1 \mu \mathrm{m}$.

Micromagnetic simulations of the trilayer reveal that the observed reversed domains in the top NiFe layer are linked to Bloch point propagation in the central $\mathrm{NdCo}$ layer. Figure 3(a) shows a snapshot of a reversal process starting from a dislocation with a black central stripe $\left(-\mathrm{M}_{\mathrm{z}}\right)$ in which a $\mathrm{Q}=-1$ head-to-head $\mathrm{BP}^{25}$ has moved along the upper dislocation branch to the left (at the location indicated by the double triangle), leaving behind a $\mathrm{Q}=1 / 2$ meron localized at the dislocation core (curved arrow). ${ }^{10}$ However, due to
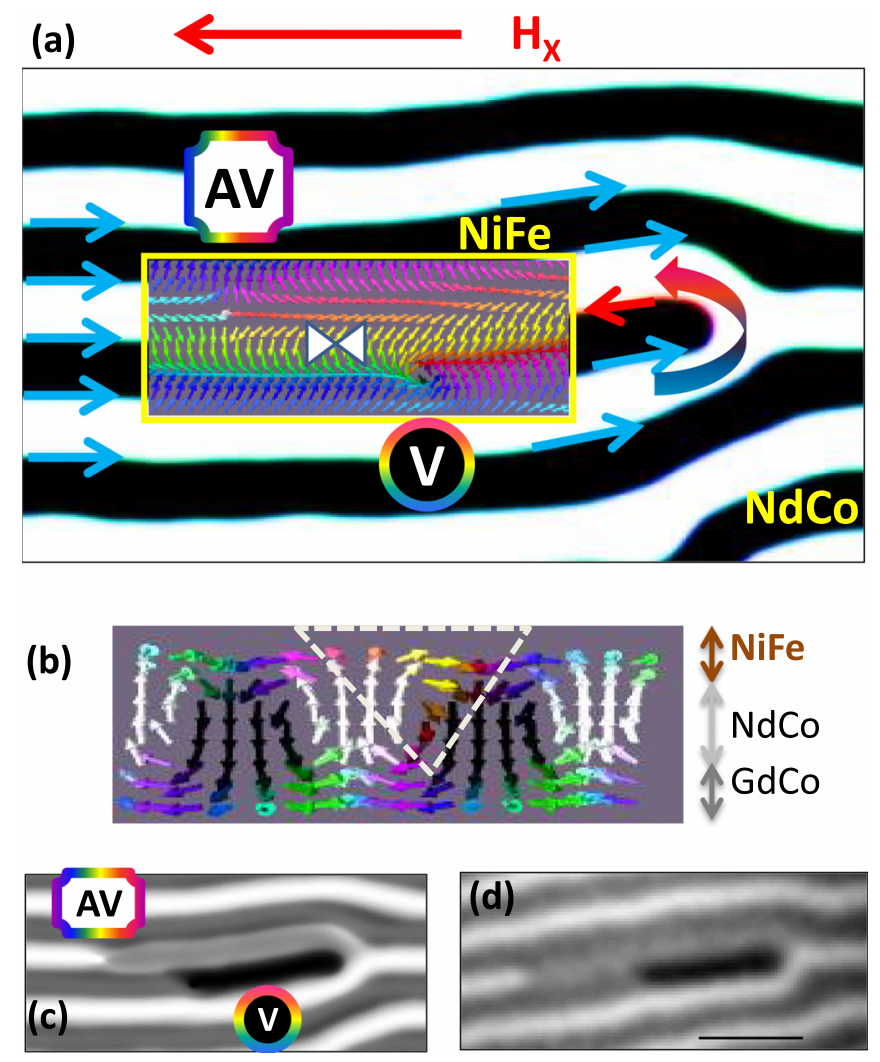

FIG. 3. (a) Micromagnetic simulation of magnetization reversal in a $40 \mathrm{~nm}$ $\mathrm{NiFe} / 60 \mathrm{~nm} \mathrm{NdCo} / 40 \mathrm{GdCo} \mathrm{nm}$ trilayer: the inset shows a vortex-antivortex pair at the NiFe surface, and the large panel corresponds to the central NdCo layer with up(white)/down(black) stripe domains that guide the V-AV propagation. In-plane reversal in the central layer occurs by the propagation of a Bloch point away from the dislocation along the boundary between up/down domains (it is located behind the inset at the position indicated by the double triangle). The curved arrow indicates a meron at the dislocation core. (b) Cross-section (y-z) of a reversed domain across the trilayer thickness. Note that it is localized in the dashed triangle near the top sample surface. (c) Simulated X-ray microscopy image of the NiFe layer from the micromagnetic configuration in (a). (d) Experimental image of a reversed domain in the NiFe layer. The scale bar is $0.5 \mu \mathrm{m}$.

asymmetries in the closure magnetization pattern, the reversal is not homogeneous across the trilayer thickness ${ }^{19}$ and, for the dislocation in Fig. 3, the reversed domain is localized near the top sample surface, i.e., mainly within the NiFe layer [see the dashed triangle in Fig. 3(b)]. A closer look at the propagating domain wall at the top surface [inset in Fig. 3(a)] reveals that the BP is dissociated into a vortex with negative polarity $\left(-\mathrm{M}_{\mathrm{z}}\right.$ core $)$ and an antivortex with positive polarity $\left(+\mathrm{M}_{\mathrm{z}}\right.$ core). $\mathrm{V}$ and $\mathrm{AV}$ are located along the lines of maximum $\mathrm{M}_{\mathrm{x}}$ which in the top layer lie just on top of the black $\left(\mathrm{M}_{\mathrm{z}}<0\right)$ and white $\left(\mathrm{M}_{\mathrm{z}}>0\right)$ domains, respectively. Therefore, they have opposite polarities, which correspond to $\mathrm{Q}=-1$, identical to that of the BP in the central plane. ${ }^{1}$ Figure 3(c) shows a calculated X-ray magnetic contrast image of the NiFe layer simulated in Fig. 3(a) that compares well with the experimental image [Fig. 3(d)]. In both cases, there is a clear difference between the reversed branch and the central stripe domain lengths attributed to the dissociated V-AV pair.

The observed configuration is similar to V-BP-AV structures reported in polarity switching processes of magnetic vortices in permalloy dots, ${ }^{2}$ but, in the present case, the 
propagation is in-plane (different from the propagation across the thickness in nanodots ${ }^{2}$ ). Also, it is interesting to note that, in spite of their opposite polarities, these $\mathrm{V}-\mathrm{AV}$ pairs propagate along linear distances of several microns guided by the stripe pattern of the trilayers, whereas in single layer permalloy nanowires, linear propagation is restricted to the same polarity pairs over hundredths of nm. ${ }^{8}$

The topological characteristics of dislocations and the structure of closure domains impose strong restrictions to the nucleation of reversed domains: first, in-plane reversal in the central layer by the propagation of a BP away from the dislocation core [dotted arrows in Fig. 4(a)] implies the reversal of the two adjacent $\mathrm{M}_{\mathrm{X}}$ lines in the top layer [continuous horizontal arrows in Fig. 4(a)], one on top of the central stripe and the other on top of the bifurcated branch. This results in the dissociation of the BP into a pair of magnetic textures (i.e., the V-AV pair). Second, for positive $\mathrm{M}_{\mathrm{X}}$ remanence, reversed domains can only appear on the top $\mathrm{NiFe} \mathrm{layer}^{19}$ at dislocations with either a black central stripe pointing to the right [Fig. 4(a)] or a white central stripe pointing to the left [Fig. 4(b)]. This selection rule, fulfilled in $100 \%$ of the experimental cases, ${ }^{19}$ also restricts the possible V-AV pairs that can propagate in the NiFe layer. For the dislocation sketched in Fig. 4(a), $M_{x}$ reversal implies the propagation of head-to-head magnetic textures towards the left of the image. Also, since it is a white bifurcation/black central stripe dislocation, $\mathbf{M}_{\mathrm{y}}$ closure domains at the top surface point towards the central stripe [vertical arrows in Fig. 4(a)]. Therefore, in the magnetic texture localized at the central stripe, magnetization points inwards at all in-plane directions (i.e., it is a radial inward vortex with negative polarity), whereas for the magnetic texture at the reversed bifurcation branch, the magnetization points inwards along $x$ and outwards along $y$ (i.e., it is an AV with positive polarity). The other possible dislocation geometry is sketched in Fig. 4(b), and we find again a $\mathrm{V}$ propagating along the central stripe and an AV at the reversed branch but with reversed polarities. In the micromagnetic simulations of Fig. 3, these purely radial vortices sketched in Fig. 4 decay into helical vortices due to the effect of exchange and in-plane anisotropy but keeping the same chirality and polarity. The propagation of V-AV pairs at top/ bottom sample surfaces has also been observed in micromagnetic simulations of magnetization reversal of the bottom GdCo layer $\left(\mathrm{K}_{\mathrm{u}}=4.6 \times 10^{3} \mathrm{~J} / \mathrm{m}^{3}\right.$ and $\left.\mathrm{K}_{\mathrm{N}}=0\right)$ in $\mathrm{NiFe} / \mathrm{NdCo} /$ GdCo trilayers and in single NdCo layers $\left(\mathrm{K}_{\mathrm{u}}=0\right.$ and $\mathrm{K}_{\mathrm{N}}=10^{5} \mathrm{~J} / \mathrm{m}^{3}$ ). This indicates that this reversal mechanism
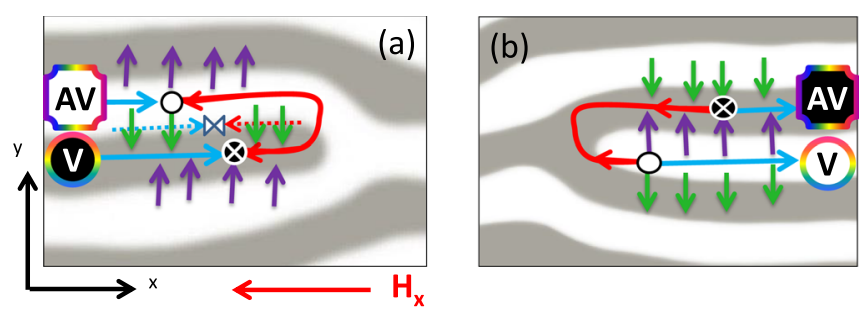

FIG. 4. (a) and (b) Sketch of the closure domain structure (continuous arrows) and $\mathrm{V}$-AV pairs at allowed dislocations on the top NiFe layer with a $\mathrm{V}$ propagating always along the central stripe and an AV at the bifurcation branch with opposite polarity $(\mathrm{Q}= \pm 1)$. Dotted arrows in (a) sketch the propagation of a BP (double triangle) in the central layer and the reversal of in-plane magnetization in the central layer. is a general feature of the stripe domain pattern in wPMA materials and does not depend on the particular anisotropy values of the chosen materials for each layer.

The experimental features of reversed domain expansion observed in Fig. 2 are in agreement with a simplified model of $\mathrm{V}$-AV pair propagation under a lateral confining potential ${ }^{4}$ that predicts faster propagation of $\mathrm{AV}$ than $\mathrm{V}$ [AV in the bifurcation branch reverses ahead of $\mathrm{V}$ at the central stripe as seen in Fig. 2(c)] and a linear increase in V-AV separation as a function of the applied field [see the increasing distance $v s$. field trend in Fig. 2(d)]. Therefore, due to the dislocation and stray field geometry, the in-plane $\mathrm{H}_{\mathrm{x}}$ separates vortices of opposite polarities towards opposite $x$ senses. This effect is very robust as it is protected by long range order in the stripe pattern, and it could be of use in vortex propagation memory schemes.

In summary, we have reported a detailed microscopic understanding of the magnetization reversal of magnetic multilayer structures combining wPMA materials with soft magnetic layers. The stripe domain pattern in the wPMA central layer is used to control the nucleation and propagation of magnetic textures in the top permalloy layer. Preferred nucleation of reversed domains occurs at dislocations within the stripe domain pattern. Inversion in the top $\mathrm{NiFe}$ layer proceeds by the creation of a vortex located in the central stripe and an antivortex located in a bifurcation branch with opposite polarities $(\mathrm{Q}=-1)$. V and AV exhibit a correlated motion along the magnetic stripes extending up to $5 \mu \mathrm{m}$, with increasing separation with the field intensity reaching ca. $1 \mu \mathrm{m}$ for fields of $25 \mathrm{mT}$. Due to topological restrictions in magnetization reversal at dislocation cores, vortices of opposite polarities are driven towards opposite $x$ senses by the in-plane field. Our findings pave the way for controlling the dynamics of magnetic singularities, which might be of use in the transport of magnetic information.

See supplementary material for a description of the calculation method of X-ray magnetic microscopy images from micromagnetic simulations.

This work was supported by Spanish MINECO [Grant Nos. FIS2013-45469 and FIS2016-76058 (AEI/FEDER, EU)] and FICYT-Asturias (FC-GRUPIN14-040). A. HierroRodriguez acknowledges support from FCT of Portugal (Grant No. SFRH/BPD/90471/2012). We thank J. Avila (Alba staff) for the pulsed magnetic field set-up.

${ }^{1}$ S. Komineas, Phys. Rev. Lett. 99, 117202 (2007).

${ }^{2}$ M. Noske, H. Stoll, M. Fähnle, R. Hertel, and G. Schütz, Phys. Rev. B 91, 014414 (2015).

${ }^{3}$ T. Devolder, J. V. Kim, M. Manfrini, W. Van Roy, L. Lagae, and C. Chappert, Appl. Phys. Lett. 97, 072512 (2010).

${ }^{4}$ D. J. Clarke, O. A. Tretiakov, G.-W. Chern, Y. B. Bazaliy, and O. Tchernyshyov, Phys. Rev. B 78, 134412 (2008).

${ }^{5}$ L. Heyne, J. Rhensius, Y.-J. Cho, D. Bedau, S. Krzyk, C. Dette, H. S. Korner, J. Fischer, M. Laufenberg, D. Backes, L. J. Heyderman, L. Joly, F. Nolting, G. Tatara, H. Kohno, S. Seo, U. Rüdiger, and M. Kläui, Phys. Rev. B 80, 184405 (2009).

${ }^{6}$ A. Ruotolo, V. Cros, B. Georges, A. Dussaux, J. Grollier, C. Deranlot, R. Guillemet, K. Bouzehouane, S. Fusil, and A. Fert, Nat. Nanotechnol. 4, 528 (2009).

${ }^{7}$ M. Manfrini, J.-V. Kim, S. Petit-Watelot, W. Van Roy, L. Lagae, C. Chappert, and T. Devolder, Nat. Nanotechnol. 9, 121 (2014). 
${ }^{8}$ S. Zhang, A. A. Baker, S. Komineas, and T. Hesjedal, Sci. Rep. 5, 15773 (2015).

${ }^{9}$ S. A. Montoya, S. Couture, J. J. Chess, J. C. T. Lee, N. Kent, D. Henze, S. K. Sinha, M.-Y. Im, S. D. Kevan, P. Fischer, B. J. McMorran, V. Lomakin, S. Roy, and E. E. Fullerton, Phys. Rev. B 95, 024415 (2017).

${ }^{10}$ C. Blanco-Roldán, C. Quirós, A. Sorrentino, A. Hierro-Rodríguez, L. M. Álvarez Prado, R. Valcárcel, M. Duch, N. Torras, J. Esteve, J. I. Martín, M. Vélez, J. M. Alameda, E. Pereiro, and S. Ferrer, Nat. Commun. 6, 8196 (2015).

${ }^{11}$ A. Hierro-Rodriguez, R. Cid, M. Vélez, G. Rodriguez-Rodriguez, J. I. Martín, L. M. A. Prado, and J. M. Alameda, Phys. Rev. Lett. 109, 117202 (2012).

${ }^{12}$ A. Hubert and R. Schäfer, Magnetic Domains: The Analysis of Magnetic Nanostructures (Springer, 1998).

${ }^{13}$ A. Hierro-Rodriguez, M. Vélez, R. Morales, N. Soriano, G. RodríguezRodríguez, L. M. Álvarez-Prado, J. I. Martín, and J. M. Alameda, Phys. Rev. B 88, 174411 (2013).

${ }^{14}$ A. Hierro-Rodriguez, J. M. Teixeira, M. Velez, L. M. Alvarez-Prado, J. I. Martın, and J. M. Alameda, Appl. Phys. Lett. 105, 102412 (2014).

${ }^{15}$ V. Vlasko-Vlasov, U. Welp, G. Karapetrov, V. Novosad, D. Rosenmann, M. Iavarone, A. Belkin, and W.-K. Kwok, Phys. Rev. B 77, 134518 (2008).

${ }^{16}$ C. Di Giorgio, F. Bobba, A. M. Cucolo, A. Scarfato, S. A. Moore, G. Karapetrov, D. D'Agostino, V. Novosad, V. Yefremenko, and M. Iavarone, Sci. Rep. 6, 38557 (2016).
${ }^{17}$ G. Siracusano, R. Tomasello, A. Giordano, V. Puliafito, B. Azzerboni, O. Ozatay, M. Carpentieri, and G. Finocchio, Phys. Rev. Lett. 117, 087204 (2016).

${ }^{18}$ D. A. Gilbert, B. B. Maranville, A. L. Balk, B. J. Kirby, P. Fischer, D. T. Pierce, J. Unguris, J. A. Borchers, and K. Liu, Nat. Commun. 6, 8462 (2015).

${ }^{19}$ A. Hierro-Rodriguez, C. Quirós, A. Sorrentino, C. Blanco-Roldán, L. M. Alvarez-Prado, J. I. Martín, J. M. Alameda, E. Pereiro, M. Vélez, and S. Ferrer, Phys. Rev. B 95, 014430 (2017).

${ }^{20}$ C. Blanco-Roldán, Y. Choi, C. Quirós, S. M. Valvidares, R. Zarate, M. Vélez, J. M. Alameda, D. Haskel, and J. I. Martín, Phys. Rev. B 92, 224433 (2015).

${ }^{21}$ E. Pereiro, J. Nicolas, S. Ferrer, and M. R. Howells, J. Synchrotron Radiat. 16, 505 (2009).

${ }^{22}$ A. Sorrentino, J. Nicolás, R. Valcárcel, F. J. Chichón, M. Rosanes, J. Avila, A. Tkachuk, J. Irwin, S. Ferrer, and E. Pereiro, J. Synchrotron Radiat. 22, 1112 (2015).

${ }^{23}$ A. Vansteenkiste, J. Leliaert, M. Dvornik, M. Helsen, F. Garcia-Sanchez, and B. Van Waeyenberge, AIP Adv. 4, 107133 (2014).

${ }^{24}$ G. E. Rowland, see http://www.grahamerowlands.com/main/muview/ for Muview.

${ }^{25}$ S. K. Kim and O. Tchernyshyov, Phys. Rev. B 88, 174402 (2013); M. Labrune and J. Miltat, J. Appl. Phys. 75, 2156 (1994). 